\title{
La souveraineté alimentaire ou le droit des peuples à décider de leurs politiques agricoles
}

Agathe DÉCARSIN

\section{(2) OpenEdition}

\section{Journals}

Édition électronique

URL : https://journals.openedition.org/ideas/538

DOI : $10.4000 /$ ideas.538

ISSN : 1950-5701

Éditeur

Institut des Amériques

Référence électronique

Agathe DÉCARSIN, «La souveraineté alimentaire ou le droit des peuples à décider de leurs politiques agricoles », IdeAs [En ligne], 3 | 2012, mis en ligne le 13 décembre 2012, consulté le 18 octobre 2022. URL : http://journals.openedition.org/ideas/538 ; DOI : https://doi.org/10.4000/ideas.538

Ce document a été généré automatiquement le 18 octobre 2022

\section{(c)}

Creative Commons - Attribution - Pas d'Utilisation Commerciale - Pas de Modification 4.0 International - CC BY-NC-ND 4.0

https://creativecommons.org/licenses/by-nc-nd/4.0/ 


\title{
La souveraineté alimentaire ou le droit des peuples à décider de leurs politiques agricoles
}

\author{
Agathe DÉCARSIN
}

1 En 2008 la hausse des prix des matières premières agricoles entrainait des émeutes de la faim et provoquait une crise alimentaire mondiale augmentant considérablement le nombre de personnes souffrant de la faim dans le monde et laissant envisager une pénurie durable des denrées alimentaires. Quelques mois plus tard, le cours des matières premières redescendit laissant entrevoir une sortie de crise et on évinça cette perspective catastrophique. Néanmoins, aujourd'hui de nombreux économistes alertent sur l'éventualité d'une nouvelle crise alimentaire provoquée par la volatilité des prix ${ }^{1}$ et cela en dépit des innovations techniques et de l'industrialisation de l'agriculture. Ces évènements récents ont montré que les marchés mondiaux orchestrés par les organismes internationaux tels que l'Organisation Mondiale du Commerce (OMC) ou la Banque Mondiale (BM) pouvaient être la source de l'insécurité alimentaire mondiale. L'OMC place les politiques de libre-échange et la concurrence des économies comme priorités pour atteindre la sécurité alimentaire ; alors que les économies locales et la production alimentaire autosuffisante sont mises à mal par ces pratiques néolibérales qui nuisent à la sécurité alimentaire des populations locales. Les limites d'un système régi par les marchés mondiaux poussent à la recherche de solutions durables afin d'assurer la sécurité alimentaire au niveau mondial.

2 La souveraineté alimentaire, principe alternatif au mouvement néolibéral, développé en 1996 par le mouvement paysan international, la Via Campesina, désigne « le droit des populations, de leur pays ou Unions à définir leur politique agricole et alimentaire, sans dumping vis-à-vis des pays tiers $»^{2}$. Selon la Via Campesina, la souveraineté alimentaire n'est pas une construction intellectuelle universitaire, il s'agit d'un " concept-action " c'est-à-dire d'une dynamique issue de réalités locales et d'un processus de transformation sociale à forte portée politique. En ce sens la souveraineté alimentaire se distingue de la notion de sécurité alimentaire définit par l'Organisation des Nations 
Unies pour l'Alimentation et l'Agriculture (FAO) comme l'accomplissement de « l'accès à une alimentation suffisante, sûre et nutritive pour toutes les personnes ». Et dénonce les limites des politiques néolibérales pour assurer un accès à une alimentation saine et nutritive ainsi que les pratiques de l'agriculture industrielle et des multinationales.

3 Selon La Via Campesina la souveraineté alimentaire est «le droit des peuples à une alimentation saine et culturellement appropriée produite avec des méthodes durables $»^{3}$, c'est pourquoi elle est reconnue au niveau international comme un principe de défense du droit à l'alimentation, droit énoncé dans la Déclaration Universelle des Droits de l'Homme de 1948 et le Pacte International relatif aux droits économiques sociaux et culturels (PIDESC) entré en vigueur en 19764. Afin d'harmoniser leur discours et de faciliter le dialogue avec les organisations institutionnelles et en particulier la FAO, les Organisations Non Gouvernementales (ONG) et les mouvements sociaux se sont réunis au sein du Comité international de planification pour la souveraineté alimentaire (CIP). Ainsi elles ont pu obtenir 4 sièges au sein du Comité de la Sécurité Alimentaire Mondiale (CSA) au même titre que les gouvernements dans le but de créer un échange constructif avec les instances politiques internationales.

Comme nous l'avons mentionné auparavant la souveraineté alimentaire s'érige en opposition aux politiques néolibérales portées par l'OMC et le processus de privatisation des biens communs par les sociétés transnationales. Au Mexique depuis la signature de l'Accord de Libre échange Nord Américain (ALENA) avec les Etats-Unis et le Canada, les multinationales de l'industrie agro-alimentaire ont le vent en poupe. Seulement 20 firmes multinationales mexicaines et étrangères sont responsables de la majeure partie de la production agroalimentaire du Mexique. Par ailleurs les importations de denrées alimentaires ont considérablement augmenté, notamment pour les matières premières comme le blé ou le maïs, céréales à la base de l'alimentation des mexicains. Ces importations de céréales issues de l'agriculture productiviste viennent concurrencer l'agriculture paysanne mexicaine et en ce sens porte atteinte au principe de souveraineté alimentaire ${ }^{5}$.

5 En effet la souveraineté alimentaire place en priorité la production agricole locale pour nourrir la population, l'accès des paysans à la terre et aux ressources naturelles ainsi qu'aux semences et au crédit et met en lumière le droit des paysannes et des paysans à produire et le droit des consommateurs à décider ce qu'ils veulent consommer.

6 Ce concept met en avant le rôle essentiel que joue l'agriculture familiale et paysanne pour nourrir la planète et lutter contre la faim et la malnutrition. Ces termes d'agriculture familiale et paysanne renvoient à un type d'agriculture diversifiée - en opposition à l'agriculture industrielle qui tend vers une production en monoculture vouée à l'exportation - permettant de nourrir à la fois le cercle familial et également d'alimenter les marchés locaux. Si ces termes sont souvent employés par les organisations de défense de la souveraineté alimentaire, il faut souligner que l'agriculture familiale et paysanne renvoie aussi bien à l'agriculture durable, à l'agroécologie ou même à l'agriculture biologique. Il s'agit d'un type d'agriculture se nourrissant à la fois des savoirs traditionnels et des innovations écologiques récentes.

7 Si chaque jour 925 millions de personnes souffrent de la faim, la cause n'est pas paradoxalement un manque de nourriture, mais un accès limité à cette nourriture pour les populations les plus pauvres. Parmi ces 925 millions de personnes, $70 \%$ vivent en milieu rural, c'est-à-dire là où sont produits les aliments. Cette situation absurde est due à une hausse constante des prix des denrées alimentaires disponibles sur le 
marché. Une des solutions à ce problème est la valorisation et le soutien à l'agriculture familiale et paysanne. En effet, on estime que si l'agriculture familiale et paysanne bénéficiait d'aides publiques suffisantes, elle pourrait offrir aux populations locales un meilleur accès aux denrées alimentaires notamment en leur procurant des denrées alimentaires de base. C'est l'idée défendue par un rapport de 2011 publié par le bureau du rapporteur spécial au droit à l'alimentation, intitulé "Agroécologie et droit à l'alimentation ${ }^{6}$, qui présente les pratiques agroécologiques comme une solution efficace à la sécurité alimentaire mondiale et propose de transposer ce modèle à plus grande échelle. Il est explicitement énoncé dans ce rapport que l'agro-écologie est créatrice d'emplois dans les milieux ruraux, massivement touchés par le chômage puisqu'elle emploie une main d'œuvre importante et en ce sens permet de ralentir l'exode rural. De même l'agro-écologie augmente la fertilité des sols et ainsi la productivité, par conséquence elle permet aux paysans de moins dépendre des subventions d'états pour se procurer des intrants, comme les engrais ou les semences améliorées. En ce sens l'agro-écologie contribue à la souveraineté alimentaire puisqu'elle donne la possibilité aux populations de maitriser leurs productions et d'avoir un meilleur accès à la nourriture.

8 Cependant l'agriculture familiale et paysanne aux méthodes respectueuses de l'environnement est mise à mal par l'agrobusiness qui vient lui faire concurrence. C'est en ce sens que le principe de la souveraineté alimentaire s'oppose aux pratiques des multinationales qui viennent accaparer des terres aux petits exploitants agricoles, pour mettre en place des monocultures peu durables et mettent en danger les droits fonciers des populations locales qui ne peuvent plus produire sur leurs propres terres. Or, le droit à la terre est étroitement lié au droit à l'alimentation. Sans terre on ne peut produire ses propres denrées alimentaires ou en tirer un revenu décent.

9 L'accès à la terre et aux ressources (eau, minéraux, semences, etc.) pour les paysannes et paysannes est une condition sine qua none pour atteindre l'objectif de la souveraineté alimentaire. Dans cette perspective, les organisations de soutien aux paysans et paysannes des pays dits du Sud et de l'Est s'attachent à lutter contre le phénomène d'accaparements de terres, qui consiste aux rachats de terres agricoles par des Etats ou des entreprises. Les causes de ce phénomène, qui ne cesse de se développer, sont diverses: premièrement, on constate une tendance, des grandes entreprises ou des Etats des pays où les terres arables ne sont pas suffisantes ou saturées par une demande croissante, à délocaliser vers l'étranger leur production alimentaire ou pour produire des agrocarburants; deuxièmement, la spéculation sur les terres par les fonds d'investissements est devenue monnaie courante dans le contexte de la crise financière qui fait miroiter une augmentation des prix du foncier dans le futur; troisièmement, certains Etats des pays dits du Sud n'hésitent pas à vendre leurs terres à des compagnies privées afin de profiter des bénéfices de ses ventes, mais à court terme. En publiant un rapport intitulé Main basse sur les terres agricoles en pleine crise alimentaire et financière ${ }^{7}$ en 2008 , l'ONG Grain a mis en lumière ce phénomène d'accaparements de terres en dénonçant la concentration des terres fertiles aux mains de quelques Etats et entreprises aux détriments des petits exploitants agricoles du monde rural. Un mois plus tard, en novembre 2008 le Financial Times dévoilait l'accaparement de 1,3 millions d'hectares de terres pour la production d'huile de palme et de maïs, par l'entreprise coréenne Daewoo à Madagascar, pays largement touché par la malnutrition et dont la population vit à $70 \%$ sous le seuil de pauvreté ${ }^{8}$. Ce coup médiatique exposa le 
phénomène d'accaparements de terres et devint un exemple emblématique de la question.

La question des accaparements de terres est également associée à la production d'agrocarburants, carburants issus de matières premières agricoles, comme les plantes oléagineuses telles que l'huile de colza, de palme, ou les féculents comme le blé ou le maïs. Dans le cadre de sa politique énergétique l'Union Européenne vise à incorporer $10 \%$ d'agrocarburants dans les transports d'ici à 2020. Cet objectif ambitieux nécessite d'affecter 15 à $20 \%$ des terres arables pour la production d'agrocarburants et pousse les pays membres de l'UE à produire toujours plus d'agrocarburants et à délocaliser leur production vers des pays où l'investissement foncier est moins coûteux. On estime que c'est « l'équivalent de 4 fois la taille de la France métropolitaine qui aurait été accaparé dans ces pays, dont environ $60 \%$ pour produire des agrocarburants $»^{9}$. Les organisations de défense de la souveraineté alimentaire, mais également les ONG écologistes, s'érigent contre cette politique énergétique en dénonçant l'utilisation de produits alimentaires pour remplir les réservoirs de nos voitures et accusant la demande croissante en matières premières de faire augmenter leurs prix au niveau mondial. Par ailleurs, les écologistes reprochent aux agrocarburants de ne pas réduire nos émissions de gaz à effets de serre, mais au contraire, de contribuer à leur augmentation si on prend en compte les effets indirects liés à leur production. En effet pour cultiver des agrocarburants industriels une utilisation massive d'engrais, de pesticides et surtout de carburants fossiles pour les machines et le transport est nécessaire, ce qui a un impact négatif sur l'environnement.

11 Une des priorités de la souveraineté alimentaire et des organisations qui adhèrent au concept est la défense et le renforcement des droits des femmes paysannes. En effet, si les paysannes représentent la majorité des paysans dans les pays dit du Sud, entre 60\% et $70 \%$ parmi elles sont défavorisées, selon une étude d'ActionAid International, ${ }^{10}$ par les politiques agricoles qui traduisent une conception patriarcale de la société. En règle générale elles ne possèdent pas les terres qu'elles cultivent, ou bien elles possèdent des terres de moindre qualité souvent éloignées de leur domicile. Par ailleurs, elles ont un accès limité aux crédits et aux emprunts, ainsi qu'aux intrants, comme les engrais ou les semences améliorées; elles reçoivent moins d'aides financières du gouvernement. Les femmes paysannes sont souvent écartées des prises de décisions collectives et ils existent peu d'organisations de paysannes. Pourtant, les femmes paysannes dans les pays dits du Sud, sont souvent en charge de la production vivrière qui permet de nourrir le foyer. En ce sens, on peut dire qu'elles sont les garantes de la sécurité alimentaire de leurs propres familles. Pour les organisations de défense de la souveraineté alimentaire, il est nécessaire de mettre l'accent sur le renforcement de l'autonomisation des femmes et de leurs droits à l'accès à la terre et aux ressources afin d'améliorer la sécurité alimentaire mondiale et d'œuvrer à l'égalité femmes-hommes. 


\section{BIBLIOGRAPHIE}

\section{Rapports}

" Agro-écologie et le droit à l'alimentation », Rapport présenté à la 16ème session du Conseil des droits de l'homme de l'ONU, Mars 2011.

http://www.srfood.org/images/stories/pdf/officialreports/20110308_a-

hrc-16-49_agroecology_fr.pdf

CURTIS Mark, «Fertile Ground : How governments and donors can halve hunger by supporting small farmers ", ActionAid, avril 2010.

Congreso Internacional de Economía del Sector Agropecuario (10.: 23 y 24 de abril, 2010 : Universidad Autónoma de Ciudad Juárez, Chih.), « La continuidad de la discusión sobre soberanía alimentaria y economía del sector agropecuario en México ", Alfonso Cortazar Martínez, Benjamín Carrera Chávez y Eduardo Pérez Eguía, Ciudad Juárez, Chih., Universidad Autónoma de Ciudad Juárez, 2011.

FAO, « La volatilité des prix sur les marchés agricoles », Rome, Janvier 2011.

Grain, « Main basse sur les terres agricoles en pleine crise alimentaire et financière », Octobre 2008.

http://www.grain.org/fr/article/entries/140-main-basse-sur-les-terres-agricoles-en-pleinecrise-alimentaire-et-financiere

\section{Articles de presse}

COLLIN Philippe et al., Libération, « Le gouvernement Ayrault doit renoncer aux politiques de soutien aux agrocarburants ", 14/09/2012.

http://www.liberation.fr/terre/2012/09/14/le-gouvernement-ayrault-doit-renoncer-auxpolitiques-de-soutien-aux-agrocarburants_845817

BURGIS Tom, Jung-a Song, Oliver Christian, Financial Times, « Daewoo to cultivate Madagascar land for free ", 19/11/2008.

http://www.ft.com/cms/s/0/6e894c6a-b65c-11dd-89dd-0000779fd18c.html\#axzz26N0A1eI6

\section{Instruments légaux internationaux}

Déclaration Universelle des droits de l'Homme (1948) :

http://www.un.org/fr/documents/udhr/

Pacte International relatif aux droits économiques sociaux et culturels, Article 2, Article 11. http://www2.ohchr.org/french/law/cescr.htm 


\section{NOTES}

1. Selon la FAO, « la volatilité mesure l'ampleur et la rapidité de l'évolution du prix d'un actif sur une période donnée, comme le prix d'un produit agricole », La volatilité des prix sur les marchés agricoles, Rome, Janvier 2011.

2. Définition de la «souveraineté alimentaire» selon La Via Campesina, voir: http:// viacampesina.org/fr/

3. Site de La Via Campesina: http://viacampesina.org

4. Protocole International des droits économiques sociaux et culturels adopté en 1976 pour compléter la DUDH.

5. "La continuidad de la discusión sobre soberanía alimentaria y economía del sector agropecuario en México", Alfonso Cortazar Martínez, Benjamín Carrera Chávez y Eduardo Pérez Eguía. Ciudad Juárez, Chih., Universidad Autónoma de Ciudad Juárez, 2011.

6. Olivier de Schutter, "Agro-écologie et le droit à l'alimentation", Rapport présenté à la 16ème session du Conseil des droits de l'homme de l'ONU, Mars 2011. Consultable en ligne à l'adresse suivante : $\quad$ http://www.srfood.org/images/stories/pdf/officialreports/20110308_ahrc-16-49_agroecology_fr.pdf

7. Grain, « Main basse sur les terres agricoles en pleine crise alimentaire et financière », Octobre 2008. Disponible en ligne: http://www.grain.org/fr/article/entries/140-main-basse-sur-lesterres-agricoles-en-pleine-crise-alimentaire-et-financiere.

8. Tom Burgis, Song Jung-a, Christian Oliver, « Daewoo to cultivate Madagascar land for free », Financial Times, 19, Nov. 2008. Disponible en ligne : http://www.ft.com/cms/s/ 0/6e894c6a-b65c-11dd-89dd-0000779fd18c.html\#axzz26N0A1eI6

9. Philippe Collin et al. "Le gouvernement Ayrault doit renoncer aux politiques de soutien aux agrocarburants”, Libération, 14 sept. 2012. Disponible en ligne : http://www.liberation.fr/terre/ 2012/09/14/le-gouvernement-ayrault-doit-renoncer-aux-politiques-de-soutien-aux-

agrocarburants_845817

10. Mark Curtis, "Fertile Ground: How governments and donors can halve hunger by supporting small farmers", ActionAid, avril 2010.

\section{AUTEUR}

\section{AGATHE DÉCARSIN}

Agathe DÉCARSIN est diplômée du Master 2 Pro Relations Interculturelles et Coopération Internationale (RICI) de l'Université Charles de Gaulle/Lille 3. Email : agathepointdecarsinatgmailpointcom. 